\title{
Satisfação e qualidade de vida em psicoterapia: um estudo piloto em clínica-escola
}

\author{
Satisfaction and quality of life in psychotherapy: \\ a pilot study in a school-clinic
}

\section{Tatiane Dias Bacelar ${ }^{1}$ (c) João Guilherme Figueredo-Campos ${ }^{2}$ (]) Frederik Curcio Lopes ${ }^{3}$ (C) Jonas Jardim de Paula 4 (1)}

\begin{abstract}
1,3Faculdade Ciências Médicas de Minas Gerais (Belo Horizonte). Minas Gerais, Brasil. tatiane.bacelar@cienciasmedicasmg.edu.br, derikcurcio@gmail.com ${ }^{2}$ Autor para correspondência. Faculdade Ciências Médicas de Minas Gerais (Belo Horizonte). Minas Gerais, Brasil. camposjgf@gmail.com ${ }^{4}$ Faculdade de Ciências Médicas de Minas Gerais (Belo Horizonte), Universidade Federal de Minas Gerais (Belo Horizonte). Minas Gerais, Brasil. jonasjardim@gmail.com
\end{abstract}

\begin{abstract}
RESUMO | INTRODUÇÃO: As clínicas-escola são preconizadas pelos cursos de psicologia como um campo para a vivência e atuação prática dos acadêmicos. Diante da importância das psicoterapias como um meio de promoção qualidade de vida e satisfação com a vida, este estudo buscou verificar a influência da psicoterapia nestes construtos, realizados por acadêmicos do $10^{\circ}$ período de psicologia, em uma clínica-escola. MÉTODO: As pessoas previstas para o atendimento de psicoterapia individual, em suas diferentes abordagens, foram avaliadas em dois tempos, ao início de um semestre letivo e ao final. Foram utilizados a WHOQOL-Bref e a Escala de Satisfação com a Vida como instrumentos de mensuração. RESULTADOS: Participaram das duas avaliações 11 pessoas (60\% mulheres, Média= 41 anos). Foi realizado o teste t pareado para comparar as médias nas duas avaliações, sendo notado um aumento significativo na satisfação com a vida e nos domínios físico e psicológico, da qualidade de vida, os domínios social e ambiental mostraram uma tendência ao aumento, ainda que não significativo estatisticamente. DISCUSSÃO: A predominância de mulheres no estudo vai ao encontro do número total de pacientes atendidos pela clínica. O aumento significativo destes construtos a partir de intervenções psicológicas, corroboram com estudos de intervenção da mesma natureza. Estudos complementares acerca da influência da psicoterapia na qualidade de vida e satisfação com a vida, no contexto de clínicas-escola, podem apoiar a realização de novas investigações científicas neste contexto, algo desafiador diante das demandas inerentes ao serviço.
\end{abstract}

PALAVRAS-CHAVE: Psicoterapia. Clínica-escola. Satisfação com a vida. Qualidade de vida.

\begin{abstract}
INTRODUCTION: School clinics are recommended by psychology's degrees as a service for the experience and practical performance for students. Knowing the importance of psychotherapies as a means of promoting quality of life and satisfaction with life, this study sought to verify the influence of psychotherapy, carried out by academics from the 10th period of psychology, in these constructs. METHOD: The participants scheduled for individual psychotherapy, in their different theories, were evaluated in two stages, at the beginning of an academic semester and at the end. WHOQOL-Bref and The Life Satisfaction Scale were used as measuring instruments. RESULTS: 11 people participated in the two evaluations (60\% women, Average $=41$ years). The paired t test was performed to compare the averages in the two assessments, with a significant increase in satisfaction with life and in the physical and psychological domains of quality of life, the social and environmental domains showed a tendency to increase, although not statistically significant. DISCUSSION: The predominance of women in the study meets the total number of patients seen by the clinic. The significant increase in these constructs from psychological interventions corroborates intervention studies of the same nature. Complementary studies about the influence of psychotherapy on quality of life and satisfaction with life, in the context of school clinics, can support the realization of new scientific investigations in this context, something challenging in view of the demands inherent to the service.
\end{abstract}

KEYWORDS: Psychoterapy. School-clinic. Satisfaction with life. Quality of life.

Como citar este artigo: Bacelar, T. D., Figueredo-Campos, J. G., Lopes, F. C., \& Paula, J. J. (2020). Satisfação e qualidade de vida em psicoterapia: um estudo piloto em clínica-escola. Revista Psicologia, Diversidade e Saúde, 9(3), 327-338. http://dx.doi.org/10.17267/2317-3394rpds. v9i3.3217 


\section{Introdução}

Pode-se afirmar que a Psicoterapia se constitui como uma prática da Psicologia e como uma área do conhecimento indispensável à formação do Psicólogo. Instituída por uma pluralidade de modelos teóricos, como Humanista, Psicanálise e Terapia CognitivoComportamental (TCC) e balizados por distintos procedimentos técnicos científicos. A Psicoterapia é compreendida segundo o Conselho Federal de Psicologia (CFP) (2000) como um processo de entendimento, assimilação e intervenção empregados de forma sistematizada e metodológica em indivíduos e/ou grupos a fim de promover a saúde mental e possibilitar o enfrentamento de conflitos e possíveis transtornos psíquicos, sendo estes fundamentados cientificamente e ratificados pela prática e ética profissional. Nesta perspectiva, no contexto acadêmico, as clínicas-escola se constituem como um campo que propicia aos estudantes de psicologia o desenvolvimento do aprendizado da prática em psicoterapia, pois se caracteriza como uma das técnicas e/ou estratégias mais ensinadas e utilizadas pelos psicólogos, desde a sua graduação (CFP, 2009).

As clínicas-escolas se configuram como um serviço determinado pelas Diretrizes Curriculares Nacionais do curso de psicologia (2004) com vistas a atender a formação profissional dos estudantes, assim como o desenvolvimento de ações que promovam a articulação das áreas ensino, pesquisa e extensão. Tal perspectiva configura-se como um desafio ao longo da rotina de atividades da Clínica-Escola. A respeito ensino, os estágios têm a função do desenvolvimento das competências profissionais necessárias a formação teórico-prática dos estudantes, sob supervisão de um professor orientador especializado. Acerca da extensão, pode-se afirmar que as práticas extensionistas possibilitam prestar serviços psicológicos à comunidade, nas diversas modalidades de atendimento psicológico, fomentando as práticas interdisciplinares entre a comunidade acadêmica e a comunidade local. Por fim, a vertente pesquisa se dá através da produção de conhecimento e propagação de saberes produzidos in loco, por meio de produções científicas (Vagostello, Albuquerque, Queiroz, \& Silva, 2017).

Especificamente em relação à pesquisa são escassos os estudos analisando a influência dos atendimentos conduzidos por estudantes, ainda que tais pesquisas sejam de suma importância para orientação dos alunos, bem como promoção de discussões e reflexões a respeito da prática e outras ações. A carência de produções científicas estão relacionadas a dois principais fatores, o primeiro é a dificuldade dos profissionais em relação às contribuições científicas à prática clínica, sendo comumente considerado apenas as formações teóricas e supervisão. O segundo fator é o foco das instituições nos estudos documentais, como por exemplo a realização da caracterização dos clientes e/ou análises de prontuários (Honda \& Yoshida, 2012; Vagostello, Albuquerque, Queiroz, \& Silva, 2017).

Assim, de acordo com Brum et al. (2012) os estudos em psicoterapia são tão antigos quanto a própria prática, sendo marcados, em geral, por dois grandes modelos de investigação. No primeiro, o enfoque é dado ao processo psicoterapêutico, ou seja, parte-se do pressuposto que se trata de processo interpessoal e complexo, sendo assim busca-se compreender, no geral, como se dão as mudanças nas pessoas atendidas. $O$ estudo de caso é um delineamento de pesquisa frequente deste modelo de avaliação. Já o segundo, volta-se para os resultados, focando na análise da eficiência e eficácia desses processos, onde determinado fenômeno psicológico é testado por meio de técnicas baseada em evidências científicas.

Neste contexto, o presente estudo buscou verificar a influência dos atendimentos psicológicos, na modalidade de psicoterapia individual, realizados em uma clínica-escola de psicologia, a partir da investigação da qualidade de vida e satisfação com a vida dos pacientes atendidos ao longo de um semestre letivo. Parte-se da premissa que se trata de construtos considerados como preditores de saúde mental (Diener \& Chan, 2011; Kamenov, Twomey, Cabello, Prina, \& Ayuso-Mateos, 2016), bem como cabe ao profissional de psicologia trabalhar visando promoção a saúde e a qualidade de vida das pessoas e das coletividades (CFP, 2005).

A qualidade de vida pode ser compreendida como a percepção do indivíduo de sua condição, no meio de seu âmbito cultural e de sistema de valores em que está inserido, relacionado à suas metas, perspectivas de futuro, esquemas e concepções (Skevington \& Epton, 2018). Segundo a Organização Mundial de Saúde (OMS) trata-se de um construto, multidimensional e subjetivo, compreendido pelos domínios da saúde física, psicológica, relações sociais e meio ambiente. O domínio da saúde física refere-se a percepção do sujeito a respeito de atividades de seu cotidiano, 
dependência de medicamentos e apoio médico, dor, sono, repouso e capacidade para o trabalho. Já a psicológica, está relacionada tanto para sentimentos positivos quanto à negativos, autoestima, aprendizagem, memória, concentração, espiritualidade, crenças pessoais e religiosidade. O domínio das relações sociais, abrange o suporte social que esse indivíduo recebe, relações pessoais e atividade sexual. Já o meio ambiente, engloba aspectos de lazer, ambiente físico, como ruídos, poluição e clima, segurança física, recursos financeiros e acessibilidade (Zimpel, Panzini, Bandeira, Fleck, \& da Rocha, 2019).

Alguns estudos demonstram fortes associações entre a diminuição da percepção do sujeito a respeito da sua qualidade de vida e a presença de condições crônicas, como doenças cardíacas, limitações físicas, depressão e ansiedade (Cruz, Polanczyk, Camey, Hoffmann, \& Fleck, 2011). Assim, denotando a significância deste construto na vida das pessoas e coletividades, este tem sido utilizado como parâmetro para a avaliação de tratamentos, intervenções e desfechos clínicos em diversos contextos, por exemplo: transplantes, intervenções psiquiátricas, reabilitação física, psicoterapia, entre outros (Kamenov et al., 2016; Skevington \& Epton, 2018).

Já satisfação com a vida, refere-se à dimensão cognitiva do bem-estar subjetivo, estando relacionada também com a qualidade de vida. Trata-se do julgamento consciente que a pessoa faz acerca de seu contexto e escolhas pessoais. Portanto, evidencia como o sujeito experiencia seu contentamento numa perspectiva racional ou intelectual, sendo um construto pouco estudado no Brasil, uma vez que prioriza-se investigações acerca da dimensão emocional ou afetiva do bem-estar subjetivo (Diener \& Chan, 2011; Seyedi Asl, Sadeghi, Bakhtiari, Ahmadi, Nazari Anamagh, \& Khayatan, 2016).

Machado et al. (2018) identificaram fortes associações entre baixos níveis de satisfação com a vida à maiores graus de ansiedade e preocupações, além de interferirem em fatores como atividades de lazer, boa qualidade do sono, apoio financeiro, idade e sexo. Nesse sentido, os autores Boehm, Winning, Segerstrom e Kubzansky (2015) realizaram estudo que associa maiores níveis de satisfação com a vida a uma redução do risco de mortalidade em uma população de adultos. Estes vão de encontro com achados na literatura que apontam a este é um construto preditivo de saúde mental e de comportamentos autodestrutivos. Deste modo, apresentando a importância da avaliação desse construto como complemento significativo a avaliação da saúde no geral e de melhores prognósticos (Diener \& Chan, 2011).

A partir da compreensão dessas variáveis e suas influências na saúde mental, a psicoterapia pode ser uma das maneiras de promover maiores influências positivas. Sendo a qualidade de vida e satisfação com a vida importantes indicadores de saúde física e mental, ambas têm sido objeto de estudos que verificam a eficácia de intervenções psicológicas, tal como psicoterápica, de modo a garantir a melhor efetividade na promoção da saúde no geral das pessoas (Sampaio et al., 2020).

Assim, este estudo piloto agrega conhecimentos acerca da prática da investigação científica no contexto de clínica-escola, pois sabe-se que não existem modelos prontos para a facilitação desta prática mediante a rotina dos serviços, entretanto, acredita-se que a partir desta contribuição, outras clínicas podem ter uma compreensão mais objetiva a respeito destes procedimentos. Além disso, este projeto contribui a respeito da influência das intervenções realizadas a partir do atendimento psicológico na modalidade de psicoterapia individual, sobretudo realizada por acadêmicos que prestam serviços à pessoas inseridas em um contexto de vulnerabilidade, seja por seu contexto social, quanto por condição psicopatológica. Neste ínterim, busca-se o aprimoramento dos serviços prestados à comunidade, bem como o fomento da integração ensino, pesquisa e extensão na clínica-escola. Diante disso, como hipóteses levantadas, considera-se que haverá um aumento significativo das médias referente a qualidade de vida - independente de seus domínios, assim como na satisfação com a vida, após o período da psicoterapia realizado por acadêmicos de psicologia.

\section{Método}

\section{Procedimentos}

Trata-se de um estudo quantitativo, longitudinal, aprovado pelo comitê de ética em pesquisa (CAAE: 06375318.0.0000.5134) da Faculdade Ciências Médicas de Minas Gerais (CMMG), realizado na Clínica-Escola de Psicologia do Ambulatório Ciências Médicas, que presta serviço credenciado para o 
Sistema Único de Saúde (SUS). Os participantes foram avaliados em dois tempos distintos, sendo eles ao início do processo psicoterapêutico, em Setembro (t1) e ao final deles, em dezembro (t2) de 2019, totalizando 03 meses de atendimento. Ressalta-se que o processo de psicoterapia foi realizado por acadêmicos do $10^{\circ}$ período do curso de psicologia.

Os participantes foram convidados para o estudo com base nos dados cadastrados na clínica-escola, em uma fila de espera por ordem cronológica de encaminhamento. Estavam previstos 91 usuários para atendimento no semestre, sendo realizado contato telefônico e agendamento da primeira avaliação (t1) para aqueles que concordaram em participar do estudo. As avaliações foram conduzidas pelos próprios pesquisadores do projeto, após a assinatura do Termo de Consentimento Livre e Esclarecido (TCLE), sendo realizadas nos consultórios da clínica-escola, de forma a não alterar a programação dos atendimentos, sendo necessário o encaixe nos horários disponíveis, via agendamento prévio.

Cada usuário respondeu aos instrumentos individualmente, em salas climatizadas, que contavam com acomodações adequadas, livre de ruídos ou interrupções, sendo duração da avaliação, em média, de 40 minutos. Foram excluídos do estudo pessoas que possuíam diagnóstico de transtornos neurocognitivos, do neurodesenvolvimento e transtornos psicóticos que apresentaram sintomatologias graves, de acordo com o Manual Diagnóstico e Estatístico de Transtornos Mentais (DSM-5) da American Psychiatric Association (APA) (2013), tendo como referência os registros retrospectivos dos atendimentos nos prontuários dos participantes.

\section{Participantes}

Compareceram à primeira avaliação do estudo (t1) um total de 35 adultos, a faixa etária dos participantes variou de 20 a 70 anos ( $M=45,00 ; D P=14,11)$, sendo (83\%) do sexo feminino. A respeito do estado civil (43\%) são solteiros, (31\%) casados(as) ou possuem união estável e (26\%) divorciados e/ou separados. Quanto a variável escolaridade (37\%) possuem ensino fundamental I e II incompleto, $(40 \%)$ ensino médio completo ou incompleto e $(23 \%)$ ensino superior completo/incompleto e/ou pós graduação. Especificamente em relação à situação socioeconômica, houve a predominância das classes econômicas C1 e C2, sendo (54\%) dos participantes, enquanto para D-E (14\%), B2 (18\%) e B1 e A5 (14\%) de acordo com os Critério de Classificação Econômica Brasil (CCEB) de 2019.

\section{Instrumentos}

Foram utilizados, nas duas avaliações do estudo, os seguintes instrumentos:

Questionário sociodemográfico, com Critério de Classificação Econômica Brasil (CCEB) de 2019: desenvolvido pela Associação Brasileira das Empresas de Pesquisa baseado no Levantamento Socioeconômico realizado pelo IBOPE em 2018. O CCEB não pretende classificar a população em termos de classes sociais e sim definir exclusivamente a população em termos de classes econômicas, ao avaliar o poder de compra de famílias urbanas brasileiras possibilita categorizar a população no que diz respeito a sua capacidade de consumo, representando uma medida proxy do nível socioeconômico (Associação Brasileira de Empresas de Pesquisas [ABEP], 2019).

World Health Organization Quality of Life - versão abreviada (WHOQOL-Bref): escala desenvolvida pela Organização Mundial de Saúde (OMS) para avaliar de maneira prática e curta a noção da população a respeito da qualidade de vida. Esta é composta por 26 questões, na qual as respostas devem ser relacionadas a situações de duas semanas anteriores à avaliação, sendo duas questões sobre a auto-avaliação da qualidade de vida e 24 questões divididas em quatro domínios: físico, psicológico, relações sociais e meio ambiente (Zimpel et al., 2019).

Escala de Satisfação com a Vida (ESV): Instrumento breve, composto por cinco perguntas gerais, com a finalidade de medir a satisfação com a vida - sendo esta um parâmetro global a respeito das próprias escolhas individuais, que se dá em uma escala tipo likert, sendo 1 "Discordo plenamente" e 7 "concordo plenamente" (Seyedi Asl et., 2016)

Análise retrospectiva dos prontuários: realizada a partir do levantamento dos registros dos atendimentos psicológicos, em prontuário eletrônico da clínica-escola, com a finalidade de identificar as características diagnósticas dos participantes, tendo como referência os critérios diagnósticos do DSM - 5 e verificar o uso e tempo de tratamento medicamentoso dos participantes. 


\section{Resultados}

Dos 35 participantes da primeira avaliação, após os três meses de acompanhamento psicoterápico ocorreu a segunda avaliação (t2), participando 11 pessoas nas duas avaliações. Após a primeira avaliação foi necessária a exclusão de 8 (29\%) participantes de acordo com os critérios dos estudo. Constatou-se que 16 (45\%) interromperam o processo de Psicoterapia, por motivos individuais e, ao longo da realização da pesquisa, 3 (8\%) não compareceram a segunda avaliação. A Tabela 1 apresenta as características sociodemográficas do grupo que participou das duas avaliações.

Tabela 1. Análise descritiva das pessoas que participaram das duas avaliações

\begin{tabular}{|c|c|}
\hline Variável & Participantes das duas avaliações $(n=11)$ \\
\hline \multicolumn{2}{|l|}{ Sexo } \\
\hline Masculino & $4(36,4 \%)$ \\
\hline Feminino & $7(63,6 \%)$ \\
\hline \multicolumn{2}{|l|}{ Estado Civil } \\
\hline Solteiro(a) & $6(54,5 \%)$ \\
\hline Casado(a)/união estável & $2(18 \%)$ \\
\hline Divorciado(a)/separado(a) & $3(27,5 \%)$ \\
\hline \multicolumn{2}{|l|}{ Nível de escolaridade } \\
\hline $\begin{array}{l}\text { Analfabeto/Ensino Fundamental I incompleto }\left(1^{\circ} \text { ao } 5^{\circ}\right. \\
\text { ano) }\end{array}$ & $1(9 \%)$ \\
\hline Ensino Fundamental II incompleto $\left(6^{\circ}\right.$ ao $9^{\circ}$ ano $)$ & $2(18 \%)$ \\
\hline Ensino médio incompleto & $2(18 \%)$ \\
\hline Médio Completo & $3(27,5 \%)$ \\
\hline Superior completo & $3(27,5 \%)$ \\
\hline \multicolumn{2}{|l|}{ Classificação socioeconômica familiar - ABEP } \\
\hline Classe D-E média salarial $\mathrm{R} \$ 719,81$ & $2(18 \%)$ \\
\hline Classe C2 média salarial $\mathrm{R} \$ 1.748,59$ & $1(9 \%)$ \\
\hline Classe C1 média salarial $\mathrm{R} \$ 3.085,48$ & $2(18 \%)$ \\
\hline Classe B2 média salarial R $\$ 5.641,64$ & $5(46 \%)$ \\
\hline Classe B1 média salarial $R \$ 11.279,14$ & $1(9 \%)$ \\
\hline
\end{tabular}

Nota: ABEP: Associação Brasileira de Empresas de Pesquisa (2019) 
Os resultados mostraram que a idade das 11 pessoas que participaram das duas avaliações variou de 20 a 72 anos $(M=41,00 ; D P=16,22)$. A partir da Tabela 1 pode-se verificar a predominância do sexo feminino $(63,6 \%)$ e estado civil solteiro(a) 54,5\%. A maioria das pessoas apresentou escolaridade: ensino médio completo (27,5\%) e superior completo $(27,5)$. Acerca do nível socioeconômico houve a predominância das classes C1 (18\%) e B2 (46\%), correspondendo a uma renda familiar média de $\mathrm{R} \$ 3.085,48$ e $\mathrm{R} \$ 5.641,64$ respectivamente.

Na Tabela 2, estão demonstradas as características específicas das pessoas atendidas e seu respectivo processo de psicoterapia. Verificou-se que a maioria, 06 (54,5\%), estiveram em acompanhamento com acadêmicos orientados pelo referencial teórico Humanista-Fenomenológico. O número de atendimentos em psicoterapia variou de 08 a 22 sessões $(M=12,54 ; D P=4,25)$. A respeito do uso de medicamentos, 08 (73\%) faziam uso de psicofármacos há mais de 06 meses. A partir da análise do prontuário constatou-se que todos os participantes apresentaram características diagnósticas de acordo com o critério do DSM-5, compatíveis com transtornos depressivos, 06 (55\%) e 05 (45\%) com transtornos de ansiedade.

Tabela 2. Características dos participantes segundo o processo de Psicoterapia

\begin{tabular}{|c|c|c|c|c|}
\hline Participantes & $\begin{array}{c}\text { Abordagem } \\
\text { Psicoterapêutica }\end{array}$ & $\begin{array}{l}\text { Número de } \\
\text { Sessões }\end{array}$ & $\begin{array}{l}\text { Sintomatologia segundo } \\
\text { DSM-5 }\end{array}$ & Uso de medicamentos \\
\hline 1 & Humanismo & 9 & Transtorno de Ansiedade & Não \\
\hline \multirow[t]{2}{*}{2} & & & Transtorno Depressivo & \\
\hline & TCC & 12 & $\begin{array}{l}\text { Maior com sintomas } \\
\text { ansiosos leves }\end{array}$ & Não \\
\hline 3 & Psicanálise & 12 & Transtorno de Ansiedade & Não \\
\hline \multirow[t]{3}{*}{4} & & & Transtorno Depressivo & \\
\hline & & & Maior com sintomas & \\
\hline & Humanismo & 10 & ansiosos leves & Sim \\
\hline 5 & Psicanálise & 13 & Transtorno de Ansiedade & Sim \\
\hline 6 & Psicanálise & 12 & Transtorno de Ansiedade & Sim \\
\hline 7 & Humanismo & 8 & Transtorno Fóbico-Ansioso & Sim \\
\hline \multirow[t]{2}{*}{8} & & & Transtorno Depressivo & \\
\hline & Humanismo & 10 & Maior Leve & Sim \\
\hline \multirow[t]{2}{*}{9} & & & Transtorno Depressivo & \\
\hline & Humanismo & 11 & Maior Moderado & Sim \\
\hline \multirow[t]{2}{*}{10} & & & Transtorno Depressivo & \\
\hline & Humanismo & 19 & Maior Moderado & Sim \\
\hline \multirow[t]{2}{*}{11} & & & Transtorno Depressivo & \\
\hline & TCC & 22 & Maior Leve & Sim \\
\hline
\end{tabular}

Nota: DSM-5: Manual Diagnóstico e Estatístico de Transtornos Mentais (5ª edição, American Psychiatric Association, 2013). 
Acerca da qualidade de vida, mensurada pela WHOQOL-bref, foi verificado, por meio do teste Kolmogorov-Smirnov, que se tratou de uma distribuição normal em todos os domínios. Procedeu-se então a análise das comparações dos resultados da primeira e segunda avaliação por meio do teste pareado $t$.

Tabela 3. Análise da qualidade de vida e satisfação com a vida dos participantes antes e após o processo de psicoterapia

\begin{tabular}{|c|c|c|c|c|c|c|}
\hline Instrumento & Domínio & & Média & $\mathrm{DP}$ & $\mathrm{t}$ & $p$ \\
\hline \multirow{9}{*}{ WHOQOL-Bref } & Físico & $\mathrm{t} 1$ & 49,02 & 24,90 & $-3,488$ & $0,006 * *$ \\
\hline & & $\mathrm{t} 2$ & 66,88 & 18,63 & & \\
\hline & & $\mathrm{t} 1$ & 45,00 & 24,90 & & \\
\hline & & $\mathrm{t} 2$ & 60,45 & 18,22 & & \\
\hline & & $\mathrm{t} 1$ & 36,36 & 20,50 & & \\
\hline & Relações Sociais & & & & $-1,951$ & 0,080 \\
\hline & & $\mathrm{t} 2$ & 54,54 & 20,19 & & \\
\hline & Moin Ambiont & $\mathrm{t} 1$ & 47,16 & 14,82 & -1520 & 0160 \\
\hline & & $\mathrm{t} 2$ & 55,69 & 18,58 & & \\
\hline \multirow[t]{2}{*}{$\begin{array}{l}\text { Escala de Satisfação } \\
\text { com a vida }\end{array}$} & $\begin{array}{l}\text { Satisfação com a } \\
\text { vida }\end{array}$ & Aval 1 & 17,000 & 8,70 & $-2,310$ & $0,043^{*}$ \\
\hline & & Aval 2 & 22,82 & 8,60 & & \\
\hline
\end{tabular}

${ }^{*} \mathrm{p}<0,01 ;{ }^{\star} \mathrm{p}<0,05$ 
A partir dos dados da Tabela 3 verificou-se em relação à satisfação com a vida relatada pelas pessoas atendidas um aumento significativo após o período de psicoterapia. Acerca da qualidade de vida os resultados mostraram diferenças significativas para os domínios físico e psicológico antes e depois da psicoterapia. Os domínios meio ambiente e relações sociais, apesar de não significativa, constatou-se uma tendência de aumento, conforme mostra o Figura 1.

Figura 1. Resultados WHOQOL-bref antes (tempo 1) e pós intervenção (tempo 2) psicoterápica. Dom1: Físico; Dom2: Psicológico; Dom3: Social; Dom4: Ambiental

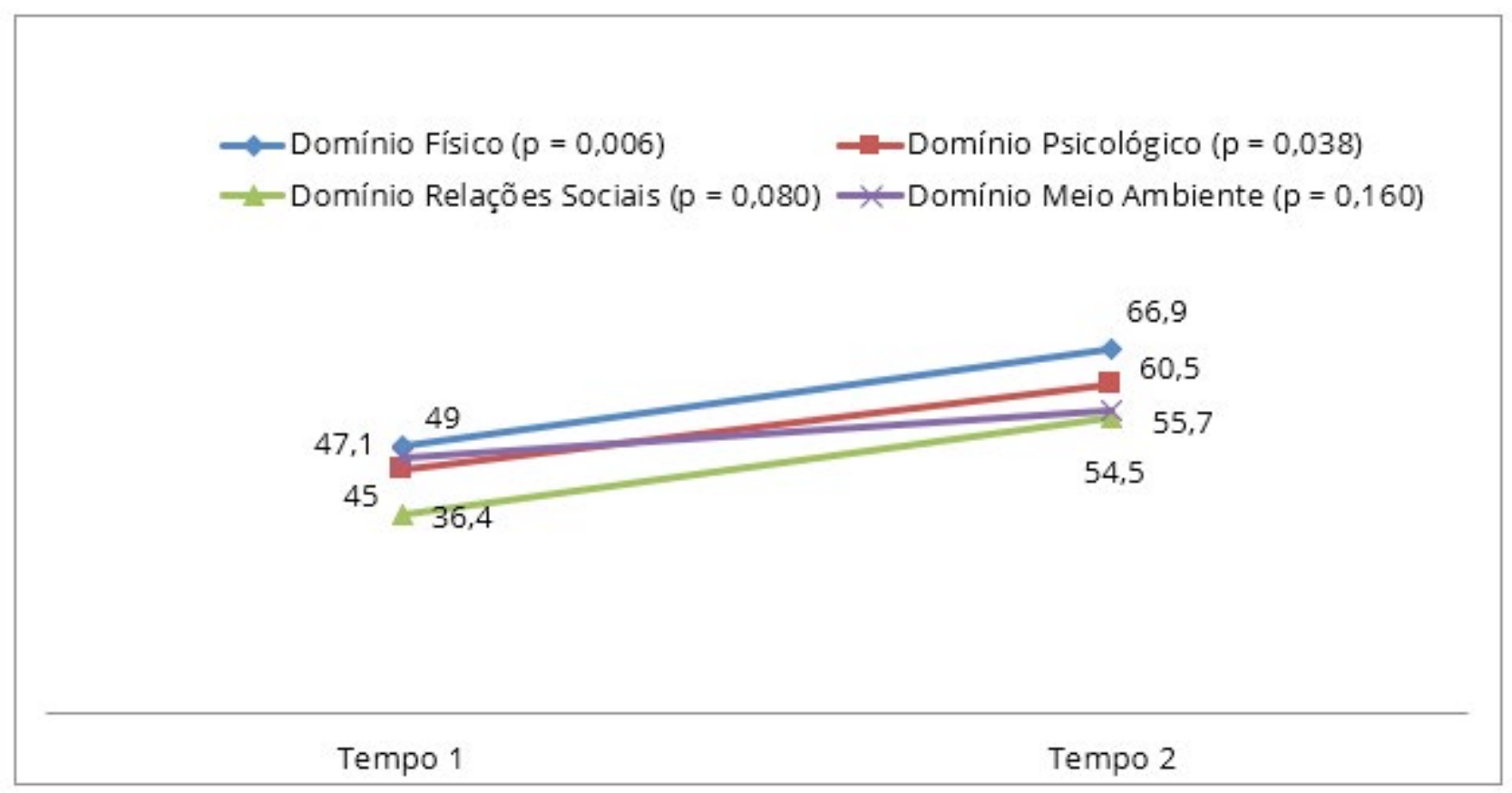

A partir do resultado que $73 \%$ dos participantes fizeram tratamento combinado de psicoterapia e psicofármacos procedeu-se a análise desta variável para verificar as diferenças na qualidade de vida e satisfação com a vida para os dois grupos, a saber: tratamento combinado e tratamento apenas com psicoterapia. Primeiramente, os escores foram submetidos ao teste, Shapiro-Wilk, para verificar a normalidade dos dados, constatando-se não se tratar de uma distribuição normal. Então, foi usado o teste de Wilcoxon para comparação do desempenho de cada um dos grupos. Os resultados mostraram haver associações significativas e positivas a favor do grupo de tratamento combinado, somente no domínio físico da WHOQOL-Bref $(p<0.036)$, sendo: a primeira avaliação (mediana $=57$, 14) e a segunda (mediana $=75,00)$. Tais associações também ocorreram em relação a satisfação com a vida ( $p<$ 0.021), sendo: a primeira avaliação (mediana $=17,00$ ) e segunda ( mediana $=26,50$ ).

\section{Discussão}

Neste estudo buscou-se verificar a influência da psicoterapia individual na qualidade de vida e satisfação com a vida das pessoas atendidas em uma clínica-escola de psicologia, ao longo de um semestre letivo. As características gerais das pessoas atendidas revelaram resultados semelhantes a outras investigações ocorridas no mesmo contexto.

Acerca das características sociodemográficas encontradas nos participantes do estudo, destaca-se que a maioria correspondeu ao sexo feminino e possuem escolaridade, ensino médio completo e ensino superior completo. Já se tratando da situação socioeconômica dos usuários, verificou-se a predominância das Classes B2 e C1. 
Neste sentido, a prevalência de pessoas do sexo feminino vai ao encontro da população feminina atendida na referida clínica-escola ao longo de 2019, representando $60 \%$ do total de pessoas atendidas, conforme o estudo realizado sobre as características sociodemográficas dos usuários (Bacelar, Silva, Campos, \& Marques, no prelo). Tal resultado também foi documentado em outros estudos realizados no mesmo contexto ao redor do país, como nos estados do Rio Grande do Sul (67,4\%), São Paulo (78,5\%) e Paraná (63,5\%) nos anos de 2003 à 2013 (Bolconte, 2014).

Acerca da predominância de indivíduos pertencentes ao nível socioeconômico médio e escolaridade média e superior, pode ser explicada pelos altos índices de desistências das pessoas localizadas na classe inferior e que possuíam poucos anos de escolarização, assim como o tamanho amostral. Em consonância com estudo realizado, Bolconte (2014) também verificou que fatores como baixa renda e baixo nível de escolaridade estão relacionados ao alto índice de abandono do tratamento psicoterapêutico em clínicas-escolas de psicologia.

Acerca da qualidade de vida, mensurada pela WHOQOL-Bref, o aumento significativo nos domínios físico e psicológico, verificado após o período da psicoterapia, também foi encontrado em outros estudos de intervenção. Em estudo longitudinal brasileiro, Sampaio et al. (2020) verificaram a eficácia da Terapia Comportamental baseada na aceitação, aplicada em contexto de grupos, no tratamento do Transtorno de Ansiedade Generalizada, e a sua resposta após três meses de intervenção. Fizeram uma escolha metodológica de mensurar sintomas ansiosos, depressivos e a qualidade de vida, não utilizando o domínio ambiental da WHOQOL-Bref em suas análises, partindo do pressuposto que as intervenções psicológicas estavam focadas nos domínios físico, psicológico e social. Assim, as evidências mostraram que após o período da psicoterapia os participantes tiveram um grande tamanho de efeito nos domínios físico e psicológico, quando comparados com o social em que houve uma pequena mudança.

Nesta direção, a prevalência do aumento significativo nos domínios físico e psicológico foi também identificado por Skevington e Epton (2018), em uma robusta meta-análise, de estudos longitudinais e interventivos (24 estudos, 11 culturas em todo o mundo), por meio da WHOQOL-Bref, constatando uma alta sensibilidade da escala para mensurar possíveis alterações, a partir de intervenções. Contudo, os autores ponderam que este fato pode estar relacionado à priorização de intervenções psicológicas focadas nestes dois domínios, em detrimento dos domínios social e ambiental.

Especificamente a respeito da relação entre qualidade de vida e transtornos depressivos, o estudo psicométrico atual da escala WHOQOL-Bref, com 404 participantes, verificou a sua validade discriminante para pessoas com diagnóstico de depressão e pessoas sem o transtorno, mensuradas a partir da Beck Depression Inventory (BDI). Constatou-se que o grupo de indivíduos com depressão apresentou uma menor qualidade de vida, em todos os domínios, principalmente no físico e psicológico (Zimpel et al., 2019). Acerca dos resultados em cada domínio, pode-se pensar que a médias do estudo psicométrico, a saber: Físico $(46,6)$, Psicológico $(55,9)$, Social, $(59,5)$ e Meio Ambiente $(51,4)$, se aproximam dos dados encontrados no presente estudo, na primeira avaliação (t1). Todavia, este estudo psicométrico não apresenta tabelas normativas, portanto outras investigações precisam ser realizadas para confirmar tal prerrogativa.

Um estudo brasileiro de normatização, com 758 participantes, também verificou diferenças significativas, com escores mais baixos em todos os domínios nas pessoas com autodeclaração de depressão, bem como de ansiedade (Cruz et al., 2011). Assim, de acordo com as médias e percentis apresentadas pelos autores em tabela normativa, os participantes do presente estudo tiveram um aumento significativo em termos de percentil, sobretudo nos domínios físico (Percentil T1=10; Percentil T2=75) e psicológico, (Percentil T1=10; Percentil T2=25). Pode-se inferir que tais mudanças são de suma importância no contexto da prática clínica em psicoterapia.

Além desta relação entre transtornos depressivos e qualidade de vida, alguns estudos têm demonstrado a influência das psicoterapias no tratamento de pessoas com diagnóstico de depressão ou apresentam uma quantidade significativa de sintomas. Sampaio, et al (2020) e Kolovos, Kleiboer, \& Cuijpers (2016) verificaram a influência positiva de intervenções psicológicas neste construto, sobretudo nos domínios físico, psicológico e social, embora neste último a mudança também foi menor em relação aos outros, corroborando os resultados aqui encontrados. 
Apesar do potencial terapêutico das psicoterapias e psicofármacos isoladamente na redução dos sintomas depressivos, as evidências têm mostrado uma maior eficiência quando as duas terapêuticas são combinadas nos tratamentos de transtornos depressivos (Kolovos, Kleiboer, \& Cuijpers, 2016). Ainda, a união destes procedimentos tem demonstrado influências significativamente positivas na qualidade de vida dos pacientes, sobretudo na percepção subjetiva a respeito deste construto, resultados que estão em consonância com o presente estudo, sobretudo na percepção do domínio físico (Kamenov, Twomey, Cabello, Prina, \& Ayuso-Mateos, 2016).

No que tange a satisfação com a vida, o aumento significativo após um período de psicoterapia, também foi verificado em estudos sobre intervenções psicológicas. Os autores Cunha, Pellanda e Reppold (2019) buscaram verificar os efeitos de intervenções focadas na gratidão em adultos e sua relação com aspectos que envolvem o bem-estar e a saúde mental, a partir da mensuração de construtos psicológicos como depressão, felicidade e satisfação com a vida. A partir da análise de 410 participantes, divididos em três grupos (foco em eventos negativos, neutros e eventos positivos), constatou-se um aumento significativo na felicidade e na satisfação com a vida, além da redução no efeito negativo e dos sintomas de depressão dos participantes que pertenciam ao grupo com intervenções focadas na gratidão.

Em outro estudo, proposto por Seyedi Asl et al. (2016), foi verificado a eficácia de intervenções baseadas na psicologia positiva e seus efeitos na satisfação com a vida, ao longo de 6 sessões. Dois grupos, controle e experimental, compostos por 18 mulheres inférteis em cada que apresentavam sintomas depressivos leves ou moderados, foram avaliados antes e após os procedimentos. Assim, constatou-se uma melhora significativa da satisfação com a vida no grupo de intervenção, não havendo mudanças no grupo controle.

Já referindo-se a estudos com enfoque interventivo que fizeram uso dos construtos qualidade de vida e satisfação com vida, por meio da WHOQOL-Bref e a Escala de Satisfação com a Vida, encontrou-se no contexto brasileiro, apenas uma pesquisa que teve como objetivo verificar a influência do Programa de Redução de Estresse, por meio da intervenção baseada em mindfulness, com duração de 6 semanas, em pessoas com sintomas depressivos e ansiosos, verificando também um aumento significativo nos domínios físico e psicológico. Nos demais domínios e na satisfação com a vida, houve uma tendência de aumento, mas não estatisticamente significativa (dos Santos et al., 2017).

As principais limitações encontradas no estudo foram: o pequeno número de amostral, a falta de um grupo controle e a utilização exclusiva do registro em prontuário para descrever a principal sintomatologia apresentada pelos participantes. Neste sentido, a predominância dos participantes realizando tratamento psicofarmacológico, simultaneamente ao andamento da psicoterapia, dificulta determinar a influência isolada dos efeitos das intervenções em psicoterapia realizada pelos estudantes na qualidade de vida e satisfação com a vida. Deste modo, um delineamento que se aproxime mais aos ensaios clínicos randomizados, estabelecendo a divisão de grupos classificados em experimental e controle, assim como o acréscimo de instrumentos que mensurem especificamente outras variáveis clínicas como sintomas de ansiedade, depressão e qualidade da relação terapêutica, poderão potencializar os resultados de novos estudos. Todavia, cabe ressaltar que trata-se um estudo piloto pioneiro na tentativa de verificar a influência das intervenções em psicoterapia realizadas nas clínicas-escola na qualidade de vida e satisfação com a vida das pessoas atendidas

Ademais, as dificuldades de realizar estudos na clínica-escola, simultaneamente a prestação de serviços à comunidade, corroboram com a carência de estudos na área. Assim, para a realização destes, torna-se necessário ajuste na logística de atendimentos, marcação de salas em períodos alternativos, conciliação de horários de avaliação da pesquisa com os horários dos pacientes, dificuldade financeiras para a locomoção dos pacientes, entre outros, visando não interferir no processo psicoterapêutico.

A partir deste levantamento pode-se perceber que embora os resultados do presente estudo estão em consonância com a literatura, grande parte dos artigos documentados sob este delineamento se distanciam do cotidiano vivenciado pelas clínicas-escola. Isto deve-se, principalmente, por ocorrerem em ambientes onde se possui o controle rígido de diversas variáveis, como número limitado de sessões, procedimentos bem delimitados e agrupamento das pessoas atendidas em grupos: controle e clínica (sem comorbidades), podendo ser pouco representativo da realidade prática. Quando transfere-se esta metodologia para o cotidiano, sobretudo em um contexto 
de clínica-escola, realizar estudos deste modelo envolve estratégias de logística interna e mudanças de rotinas, assim como um método científico adequado para sua execução (Brum et al., 2012).

\section{Considerações finais}

Os resultados deste estudo, embora piloto, apontam para a relevância da realização de investigações científicas com intuito de mensurar os desfechos dos processos interventivos realizados na modalidade de psicoterapia, mesmo diante das dificuldades inerentes a avaliação de um processo tão complexo. Tal relevância ganha força por se tratar de um contexto de clínica-escola, uma vez que, como apontado por outros autores, a realização de pesquisas impulsionam a adoção de estratégias que visam tornar a sua prestação de serviços mais eficiente e de maior qualidade.

Nesse sentido, os instrumentos utilizados, Escala de Satisfação com a Vida e a WHOQOL-bref, demonstraram possuir uma alta sensibilidade para avaliar intervenções, podendo ser recursos importantes à mensuração em diferentes contextos, como social, educacional e de saúde, tanto mental como física. Configuram-se como ferramentas úteis ao aprimoramento de estratégias de avaliação, intervenção e prognósticos, no contexto de saúde no geral. Embora a amostra tenha sido reduzida, carecido de mais instrumentos específicos de variáveis clínicas e não tenha sido realizada a separação sistemática entre grupos, como experimental e controle, os resultados indicaram mudanças significativas após o processo de intervenção psicoterápica, corroborando parcialmente com as hipóteses previamente levantadas.

Assim, neste trabalho buscou-se realizar os procedimentos de pesquisa de maneira a não impactar na rotina de atendimentos da clínica, bem como interferir nos atendimentos, uma vez que ocorreu de forma paralela ao acompanhamento psicológico. Cabe ressaltar que este trabalho fomenta discussões a respeito das dificuldades e viabilidades de investigações científicas com delineamento longitudinal no contexto de clínica-escola, na expectativa de inspirar outras instituições a produzirem, replicarem e divulgarem os saberes produzidos neste campo fértil de produção do conhecimento. Nesse sentido, novas investigações são necessárias a fim de compreender as diferentes variáveis que influenciam o processo de psicoterapia, sobretudo realizado por acadêmicos de psicologia.

\section{Agradecimentos}

Agradecemos a Faculdade Ciências Médicas de Minas Gerais por contribuir financeiramente através do Programa de Bolsas de Iniciação Científica (PROBIC) para realização deste estudo.

\section{Contribuições dos autores}

Bacelar TD foi responsável pela revisão de literatura, orientação e supervisão da escrita do projeto, participou da concepção, delineamento, análise estatística dos dados da pesquisa, interpretação dos resultados e redação do artigo científico. Figueredo-campos J. G. participou da concepção, delineamento, coleta de dados, análise estatística dos dados da pesquisa, interpretação dos resultados, redação do artigo científico. Lopes, F. C. participou da concepção, delineamento, coleta de dados, revisão de literatura, interpretação dos resultados e redação do artigo científico.

\section{Conflitos de interesses}

Nenhum conflito financeiro, legal ou político envolvendo terceiros (governo, empresas e fundações privadas, etc.) foi declarado para nenhum aspecto do trabalho submetido (incluindo, mas não se limitando a subvenções e financiamentos, participação em conselho consultivo, desenho de estudo, preparação de manuscrito, análise estatística, etc.)

\section{Referências}

Associação Brasileira de Empresas de Pesquisa (2019). Critério de classificação econômica Brasil. http://www.abep.org/ criterio-brasil

American Psychiatry Association (2013). Diagnostic and statistical manual of mental disorders (5th.ed.) American Psychiatric Publishing.

Bacelar, T. D., Alves, S. P., Campos, J. G. F., \& Marques, L. N. (no prelo). O fazer em uma clínica-escola de psicologia: os desafios da integração ensino, pesquisa e extensão. Práticas e Desafios: Estágio em Clínicas Escolas de Psicologia na Contemporaneidade. Artesã.

Boehm, J. K., Winning, A., Segerstrom, S., \& Kubzansky, L. D. (2015). Variability Modifies Life Satisfaction's Association With Mortality Risk in Older Adults. Psychological Science, 26(7), 1063-1070. https://doi.org/10.1177/0956797615581491 
Bolconte, I. S. (2014). Perfil do público das clínicas-escola de Psicologia do Brasil: Uma revisão (Trabalho de conclusão de curso, Universidade Estadual da Paraíba). https://bit. ly/2UVw1MH

Brum, E. H, M., Frizzo, G. B., Gomes, A. G., Silva, M. R., Souza, D. D., \& Piccinini, C. A. (2012). Evolução dos modelos de pesquisa em psicoterapia. Estudos de Psicologia, 29(2), 259-269. https://doi.org/10.1590/S0103-166X2012000200012

Conselho Federal de Psicologia (2005). Código de ética profissional do Psicólogo. http://site.cfp.org.br/wp-content/ uploads/2012/07/codigo-de-etica-psicologia.pdf

Conselho Federal de Psicologia (2009). Ano da psicoterapia: Textos geradores. https://site.cfp.org.br/wp-content/ uploads/2009/05/Ano-da-Psicoterapia-Textos-geradores.pdf

Conselho Federal de Psicologia, Conselho regional de Psicologia de São Paulo, \& Associação Brasileira de ensino de Psicologia (Orgs) (2013). Carta de Serviços sobre Estágio e Serviços Escolas - CFP. https://site.cfp.org.br/wp-content/ uploads/2013/09/carta-de-servicos-sobre-estagios-eservicos-escola12.09-2.pdf

Cruz, L. N., Polanczyk, C. A., Camey, S. A., Hoffmann, J. F., \& Fleck, M. P. (2011). Quality of life in Brazil: normative values for the WHOQOL-bref in a southern general population sample. Quality of Life Research, 20(7), 1123-1129. http:// dx.doi.org/10.1007/s11136-011-9845-3

Cunha, L. F., Pellanda, L. C., \& Reppold, C. T. (2019). Positive psychology and gratitude interventions: A randomized clinical trial. Frontiers in Psychology, 10. https://doi. org/10.3389/fpsyg.2019.00584

Diener, E., \& Chan, M. Y. (2011). Happy people live longer: subjective well-being contributes to health and longevity. Applied Psychology: Health and Well-Being, 3(1), 1-43. https:// doi.org/10.1111/j.1758-0854.2010.01045.x

Honda, G. C., \& Yoshida, E. M. P. (2012). Mudança em Pacientes de Clínica-escola: Avaliação de Resultados e Processos. Paidéia, 22(51), 73-82. https://doi.org/10.1590/S0103$863 \times 2012000100009$

Kamenov, K., Twomey, C., Cabello, M., Prina, A. M., \& AyusoMateos, J. L. (2016). The efficacy of psychotherapy, pharmacotherapy and their combination on functioning and quality of life in depression: a meta-analysis. Psychological Medicine, 47(3), 414-425. https://doi. org/10.1017/S0033291716002774

Kolovos, S., Kleiboer, A., \& Cuijpers, P. (2016). Effect of psychotherapy for depression on quality of life: metaanalysis. The British journal of psychiatry, 209(6), 460-468. https://doi.org/10.1192/bjp.bp.115.175059

Machado, L., Souza, C. T. N., Nunes, R. O., Santana, C. N., Araujo, C. F., \& Cantilino, A. (2018). Subjective well-being, religiosity and anxiety: a cross-sectional study applied to a sample of Brazilian medical students. Trends in psychiatry and psychotherapy, 40(3), 185-192. http://dx.doi. org/10.1590/2237-6089-2017-0070

Resolução CFP $n^{\circ}$ 10/00, de 20 de dezembro de 2000. Especifica e qualifica a Psicoterapia como prática do Psicólogo. https://site.cfp.org.br/wp-content/uploads/2000/12/ resolucao2000_10.pdf

Resolução CNE/CES N 5 de 15 de março de 2011. Diretrizes Curriculares Nacionais para os cursos de graduação em Psicologia, estabelecendo normas para o projeto pedagógico complementar para a Formação de Professores de Psicologia. Brasília, DF: Conselho Nacional de Educação.

Sampaio, T. P .A., Jorge, R.C., Martins, D.S., Gandarela, L. M., Hayes-Skelton, S., Bernik, M A., ... Lotufo-Neto, F. (2020). Efficacy of an acceptance-based group behavioral therapy for generalized anxiety disorder. Depression and Anxiety, 1-15. https://doi.org/10.1002/da.23021

Santos, T. M., Kozasa, E. H., Carmagnani, I. S., Tanaka, L. H., Lacerda, S. S., \& Nogueira-Martins, L. A. (2016). Positive Effects of a Stress Reduction Program Based on Mindfulness Meditation in Brazilian Nursing Professionals: Qualitative and Quantitative Evaluation. Explore, 12(2), 9099. https://doi.org/10.1016/j.explore.2015.12.005

Seyedi Asl, S. T., Sadeghi, K., Bakhtiari, M., Ahmadi, S. M., Nazari Anamagh, A., \& Khayatan, T. (2016). Effect of Group Positive Psychotherapy on Improvement of Life Satisfaction and The Quality of Life in Infertile Woman. International Journal of Fertility \& Sterility, 10(1), 105-112. https://10.22074/ijfs.2016.4775

Skevington, S. M., \& Epton, T. (2018). How will the sustainable development goals deliver changes in well-being? A systematic review and meta-analysis to investigate whether WHOQOL-BREF scores respond to change. BMJ Global Health, 3(Suppl 1), e000609. doi: https://10.1136/ bmjgh-2017-000609

Vagostello, L., Albuquerque, D. S. M., Queiroz, F. T., Lopes, G. P., \& Silva, L. P. (2017). Caracterização das demandas de psicodiagnóstico infantil em uma clínica-escola de São Paulo. Psicologia Revista, 26(1), 41-58. https://doi. org/10.23925/2594-3871.2017v26i1p.41-58

Yoshida, E. M. P. (2005). Recepção, acolhimento, triagem e pesquisa na clínica psicológica. In L. L. Melo-Silva, M. A. Santos, \& C. P. Simon (Orgs.), Formação em psicologia: Serviços-escola em debate (pp. 271-280). Vetor.

Zimpel, R. R., Panzini, R. G., Bandeira, D. R., Fleck, M. P., \& Rocha, N. S. (2019). Psychometric properties of the WHOQOLSRPB BREF, Brazilian Portuguese version. Brazilian Journal of Psychiatry, 41(5), 411-418. https://doi.org/10.1590/15164446-2018-0083 